\title{
Cope's Rule and the Universal Scaling Law of Ornament Complexity
}

\author{
Pasquale Raia, ${ }^{1, \star}$ Federico Passaro, ${ }^{1}$ Francesco Carotenuto, ${ }^{1}$ Leonardo Maiorino, ${ }^{2}$ Paolo Piras, ${ }^{2}$ \\ Luciano Teresi, ${ }^{3}$ Shai Meiri, ${ }^{4}$ Yuval Itescu, ${ }^{4}$ Maria Novosolov, ${ }^{4}$ Mattia Antonio Baiano, ${ }^{5}$ \\ Ricard Martínez, ${ }^{6}$ and Mikael Fortelius ${ }^{7}$
}

1. Dipartimento di Scienze della Terra, dell'Ambiente e delle Risorse (DISTAR), Università degli Studi di Napoli Federico II, Largo San Marcellino 10, 80138 Naples, Italy; 2. Dipartimento di Scienze, Università degli Studi Roma Tre, Largo San Leonardo Murialdo 1, 00146 Rome, Italy; 3. Dipartimento di Matematica e Fisica, Università degli Studi Roma Tre, Largo San Leonardo Murialdo 1, 00146 Rome, Italy; 4. Department of Zoology, Tel Aviv University, 26 Michal Street, 63261 Tel Aviv, Israel; 5. Institut Català de Paleontologia Miquel Crusafont (ICP), Carrer Escola Industrial 23, Sabadell, E-08201 Catalonia, Spain; 6. Departament de Geologia (Paleontologia), Universitat Autónoma de Barcelona, 08193 Bellaterra, Spain; 7. Department of Geosciences and Geography, Gustaf Hällströmin katu 2a, University of Helsinki, FI-00014 Helsinki, Finland

Submitted August 8, 2014; Accepted February 27, 2015; Electronically published May 29, 2015

Online enhancement: appendix. Dryad data: http://dx.doi.org/10.5061/dryad.50dr8.

\begin{abstract}
A BSTRACT: Luxuriant, bushy antlers, bizarre crests, and huge, twisting horns and tusks are conventionally understood as products of sexual selection. This view stems from both direct observation and from the empirical finding that the size of these structures grows faster than body size (i.e., ornament size shows positive allometry). We contend that the familiar evolutionary increase in the complexity of ornaments over time in many animal clades is decoupled from ornament size evolution. Increased body size comes with extended growth. Since growth scales to the quarter power of body size, we predicted that ornament complexity should scale according to the quarter power law as well, irrespective of the role of sexual selection in the evolution and function of the ornament. To test this hypothesis, we selected three clades (ammonites, deer, and ceratopsian dinosaurs) whose species bore ornaments that differ in terms of the importance of sexual selection to their evolution. We found that the exponent of the regression of ornament complexity to body size is the same for the three groups and is statistically indistinguishable from 0.25 . We suggest that the evolution of ornament complexity is a by-product of Cope's rule. We argue that although sexual selection may control size in most ornaments, it does not influence their shape.
\end{abstract}

Keywords: biological scaling, allometry, sexual selection, heterochrony, ornamental structures.

Nature offers a bewildering diversity of ornaments. The flamboyant plumage or exaggerated tails in several male birds, the conspicuous mane of male lions, the horns of rhinoceroses and stag beetles, the huge claw of male fiddler crabs, elaborate frills in ceratopsian dinosaurs, and the antlers of deer are but a few examples of this spectac-

* Corresponding author; e-mail: pasquale.raia@unina.it.

Am. Nat. 2015. Vol. 186, pp. 165-175. (C) 2015 by The University of Chicago. 0003-0147/2015/18602-55685\$15.00. All rights reserved.

DOI: $10.1086 / 682011$ ular, sometimes bizarre, diversity of ornament shapes and sizes. The evolution of these traits has always attracted considerable interest from evolutionary biologists (Darwin 1871). When only males possess ornaments (usually defined as exaggerated traits with high variability and no obvious functional explanation; Emlen 2008), they most probably function as secondary sex characteristics. This means that sexual selection could be held responsible for their evolution (West-Eberhard 1983; Andersson 1994; Møller and Birkhead 1994; Stuart-Fox and Ord 2004; Emlen et al. 2012). In fact, however, ornaments frequently appear in both sexes of the same species in many animal clades (Knell 2013), both extinct and living. Sexual selection, however, may still control the evolution of ornaments in such cases. When male parenting is costly, males, like females, have to be choosy at picking mates (Kokko and Johnstone 2002). Thus, females should compete with each other over mates, and sexually attractive ornaments would therefore develop in both sexes (Bonduriansky 2007). This form of mutual sexual selection is probably very common (Bergstrom and Real 2000). It is present in several animal clades such as insects, fishes, squamates, and birds and has been claimed to explain the presence of elaborate cranial ornaments in ornithodirans (the clade including dinosaurs and pterosaurs; Hone et al. 2012).

Although sexual selection is the most pervasive explanation for the evolution and persistence of ornaments, there are several cases of ornaments that are used or at least recruited for functions not explicitly related to mate choice. For instance, horns in females of several ungulate species may serve as antipredator devices (Caro et al. 2003). Facial ornaments may have served for species recognition in di- 
nosaurs (Padian and Horner 2011, 2014) as they do in Neotropical primates (Santana et al. 2012). Padian and Horner (2011) commented that any ornament evolving under whatever form of selection should produce a trend for increase in size and complexity over time, because the larger and more conspicuous the ornament is, the higher the fitness advantage it confers (Andersson 1994; Kuijper et al. 2012). In contrast, it is often postulated that sexual selection could initiate shape divergence among ornaments in unpredictable ways and that evolution would then proceed by increasing both the size and the shape complexity of the trait (West-Eberhard 1983). This implies that ornament shape would be subjected to sexual selection just as ornament size is, a view that is widespread in the biological literature (West-Eberhard 1983; Andersson 1994; Møller and Birkhead 1994; Stuart-Fox and Ord 2004; Emlen 2008; Emlen et al. 2012).

In sum, whereas the role of sexual selection in ornaments' existence and evolution is questionable in many cases, the evolution of their shape is unanimously seen as coupled to that of their size. We argue to the opposite. Our hypothesis is that ornament complexity (hence, shape) is not influenced by sexual selection. We contend that it could be a simple by-product of body size increase through time within clades (an ubiquitous pattern known as Cope's rule;
Stanley 1973; Raia et al. 2012). Increased body size comes with an extended ontogenetic growth period (Roff 2002). Interspecifically, this relationship is mathematically represented by the power law: growth $\approx M^{0.25}$, where growth is the time spanning from birth to adulthood and $M$ is body size. Ornaments usually become more complex during an individual's ontogeny (although there are exceptions, this is generally true of the clades we tested here; Dommergues 1990; Sampson et al. 1997; Geist 1998; Sampson 2001). Thus, if the rate of complexity increase during ontogeny holds constant from one species to its direct descendant, then the ornament of the descendant species would be $\left(M_{\text {descendant }} / M_{\text {ancestor }}\right)^{0.25}$ times more complex than its ancestor's ornament, provided that $M_{\text {descendant }}>M_{\text {ancestor }}$. This provides a testable hypothesis about how complexity should scale if all of the ornaments we tested here become more complex as a by-product of Cope's rule (Raia and Fortelius 2013; fig. 1). Importantly, under sexual selection theory, ornament complexity should scale to $>0.25$ power, since there would be positive selection for the trait complexity itself, as happens with ornament size (Andersson 1994; Emlen 2008).

The assumption that the rate of increase in complexity during ontogeny holds constant in ancestor-descendant relationships is certainly simplistic. We do not imply that

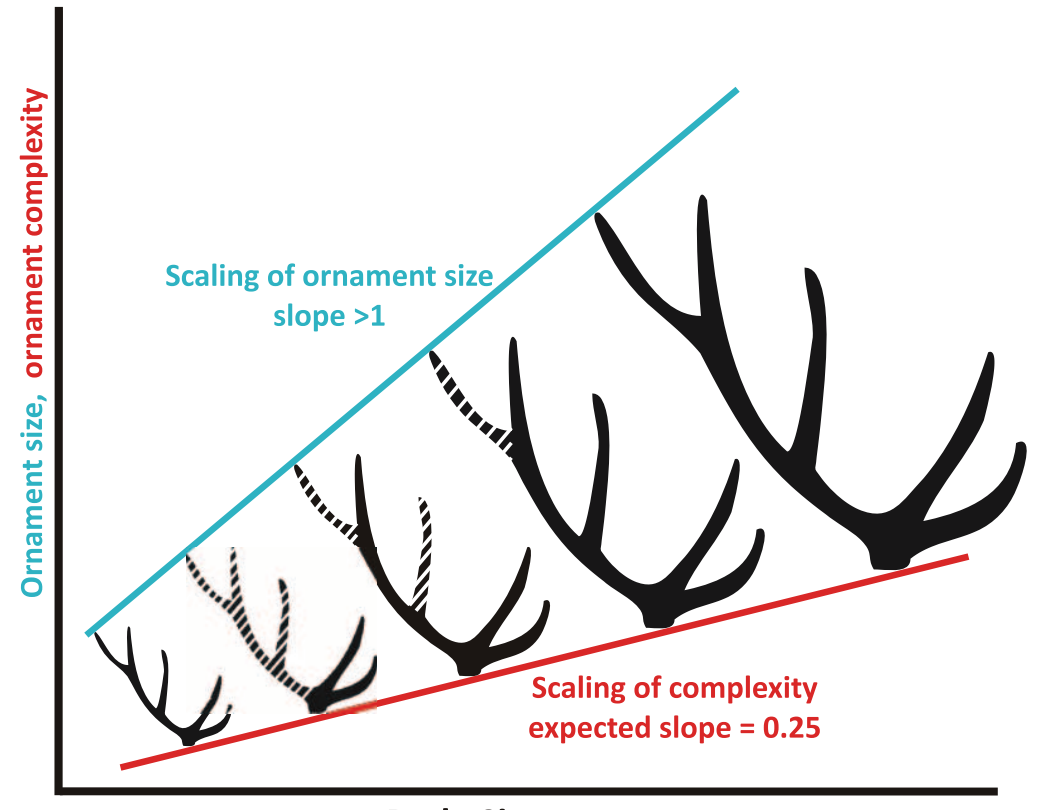

Body Size

Figure 1: Allometric scaling relationships, or ornament size and complexity, as we expected here. We used a stylized representation of deer antlers to illustrate the interspecific scaling of the ornament size (blue line) and ornament complexity (red line), according to our expectations. Our model implicitly assumes that there is a common ontogenetic shape trajectory across all the species (represented in the figure by their antlers). The solid black portion of each antler indicates the degree of completion of the ontogenetic trajectory in each species. As body size grows, antler size increases much faster than the complexity of its shape. Since complexity evolution is herein expected to be dependent only on the length of the ontogenetic growth period, its scaling exponent should be 0.25 . 
this is always true for all of the species in the clades we analyzed. There is evidence that complexity may decrease during ontogeny, even in heavily ornamented animals such as Triceratops (Horner and Goodwin 2006) or in the Dracorex/Stygimoloch/Pachycephalosaurus species group (Horner and Goodwin 2009). Thus, although our somewhat naïve assumption will certainly be met by some noise in the data, it still provides an effective means to tell apart the effect of sexual selection from Cope's rule on the scaling of ornament complexity.

Ornaments do show such a conspicuous trend for increase in size and complexity over evolutionary time in a variety of animal clades (Bonner 1988). These include at least the antlers of male deer (Geist 1998), the frill of ceratopsian dinosaurs (Dodson et al. 2004), and the suture lines in ammonoid cephalopods (Dommergues 1990). Sexual selection is obviously at play in deer antler evolution (Geist 1998), while its contribution to the evolution of ceratopsian frill is uncertain (Padian and Horner 2013). Finally, sexual selection could certainly not cause the increase in complexity through time in the ammonite suture, because sutures were covered in tissue in the living animals. Although suture lines are not proper "ornaments," we chose to study ammonites (in addition to deer and dinosaurs) exactly to test whether the evolutionary trend in ammonites differs from the trend seen in deer and, possibly, in dinosaurs, whereas we specifically contend it should be the same.

To test our hypothesis, we first prepared separate phylogenetic trees for 241 ammonite genera, 41 fossil deer species, and 34 ceratopsian species. Then we computed linear regressions between ornament complexity and body size by using both sets of raw data (allometric scaling; see "Material and Methods") in a phylogenetically controlled context, testing for different models of ornament evolution by means of phylogenetic generalized least squares (PGLS). PGLS allows testing if ornament complexity evolution is really driven by change in body size, regardless of phylogenetic effects.

For ammonites and deer, we also regressed complexity on stratigraphic duration to see whether more complex ornaments evolve with higher extinction rate, as expected under certain models of evolution of biological complexity (Stanley 1979). Species longevity in ceratopsians is not available, given that the paucity of their fossil record does not allow calculating reliable stratigraphic ranges.

\section{Material and Methods}

\section{Computation of the Fractal Dimension}

In order to numerically quantify ornament complexity in ammonites and ceratopsians, we computed the fractal di- mension of their ornaments (fig. 2). We collected reproduced images of the ammonite sutures from the literature (see appendix, available online). Each suture was placed on a white rectangular background with sides of 1,000 and 500 pixels in order to occupy most of the available space. We carefully tried to include only sutures representing the last septum of adult individuals. The suture images were cleaned so that they had a thickness of 5 pixels. We averaged fractal dimensions from different species within each genus.

For the ceratopsian frill, we followed the same basic procedure (fig. 2). However, rather than scanning the literature, we directly photographed the frills of 75 dinosaur specimens at museums we visited (see appendix). The photographs (taken dorsally, in orthogonal view) were transferred to a blank square with a white background and a 1,000-pixels side. Since several skulls are deformed on either of the two sides, we used one side only for each specimen in order to prevent distortion from affecting the calculation of the fractal dimension of the frill margin. Then, we cut pictures at the anterior-most anatomical point of the parietal bone. As the thickness of an outline can affect the computation of its fractal dimension, we overdrew the frill profile by superimposing a line 5 pixels in thickness. This line was then used to compute the fractal dimension. A number of specimens were excluded from the analyses because either they present excessive deformation or we were not confident they represent adult individuals. We averaged fractal dimensions at the species level.

Profiles were processed with the software FracLac, version 2.5 (Karperien 1999), a freeware application associated to an image processor named ImageJ (Abràmoff et al. 2004).

\section{Computation of the Strahler Number}

The Strahler number is a measure of complexity of hydrographic nets (Strahler 1957). It is computed so that highly branching streams within the net will have larger values. Deer antlers can be easily envisaged as hydrographic nets, and the Strahler number can therefore be calculated for each segment of the antler. Then, Strahler numbers are summed over the entire antler to obtain an overall estimate of its complexity (fig. 2). For instance, a simple antler with one fork, as in the muntjak Muntiacus muntjak, will have sum of Strahler numbers $\mathrm{sSN}=4$ (the beam has a value of 2 , and the two prongs have a value of 1 each; hence, $2+1+1=4)$. A three-pronged sambar deer Rusa unicolor has $\mathrm{sSN}=7$ ( 1 for each prong, plus 2 for the single fork, and 2 for the beam; hence, $2+2+1+1+1=7$ ), and so on. For each species, we computed the $(\log )$ of sSN as implemented in the R package phytools (Revell 2012). To do so, we first converted the antler shape into a phylo- 


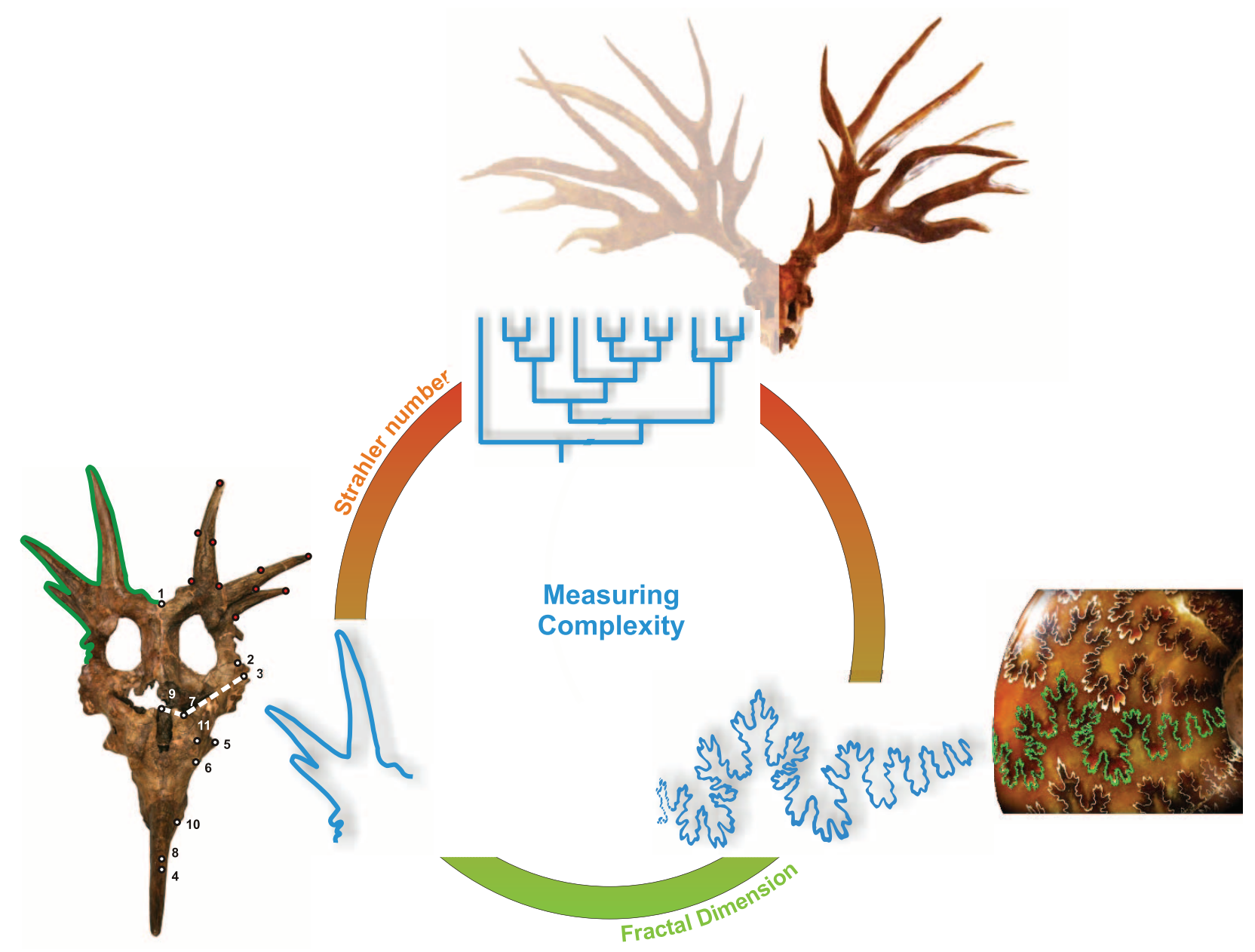

Figure 2: Schematic representation of the methods used to compute the complexity index of ornamental traits in the three different clades. For deer, we calculated the complexity of the antlers by converting them in a phylogenetic tree and then calculating the sum of the Strahler number of the tree. The skull of a male Pleistocene deer Eucladoceros dicranios (top) is portrayed as an example. For ammonites and ceratopsians, we computed the fractal dimension of the sutures (in blue) and of the frill outer margin (in green), respectively, as an index of ornament complexity. The circles (white $=$ landmarks, red $=$ semilandmarks) on the skull of Styracosaurus albertensis (bottom left) shows the configuration of landmarks used to calculate the skull centroid size in ceratopsians. The dashed white line represents the divide between the frill and the snout regions of the skull. For ammonites, we retrieved from literature the suture lines and computed their fractal dimension. A portion of the shell of the ammonite Cleoniceras (bottom right) is portrayed as an example.

genetic tree in Mesquite (Maddison and Maddison 2001). We selected adult complete antlers for each species in the tree (see appendix). We considered a typical adult antler to be one that had the highest number of tines for that species, provided at least two similarly complex antlers were observed in the fossil record (to avoid including unusual antlers with an excessive number of tines). Some deer (e.g., fallow deer, extinct Irish elk Megaloceros) have palmate antlers. In these cases, the palmation was converted (in Mesquite) in a polytomy subtending a number of species equal to the number of distinct tines present in the real antler. This probably could overestimate the $\mathrm{sSN}$ of palmateantlered deer, which tend to be more common toward the
Recent. On the other hand, we did not include species without antlers that were somewhat common during the early evolution of deer. This could very probably increase the slope of the sSN-to-body-size relationship. Hopefully, the effects of palmation treatment and discarding species without antlers cancel each other out.

\section{Body Size Data}

Body sizes for extant and extinct deer were taken from Raia et al. $(2012,2013)$ unless otherwise indicated (see appendix). For the species for which we had no body size estimates, we applied known regression equations of body 
size on length of the third lower molar (see Raia et al. 2012, 2013 for details). For ammonites, we took generawise average shell diameters. The sources for these data are indicated in the appendix.

Body size is not available for many ceratopsian species due to their fragmentary fossil material. Published cladewise data report rough estimates of total body lengths (Holtz 2007; Paul 2010) or simply do not cover enough ceratopsians (Benson et al. 2014). Therefore, we relied on the "size" of a configuration of landmarks superimposed over the skull under the geometric morphometric approach (the centroid size, CS) to get a proxy for body size. This allows estimating both a good proxy for body size and the complexity index on the same individual skull. The CS is computed as the square root of sum of squared differences between landmarks from their centroid (Bookstein 1986). The CS is routinely used as an index of "size" in geometric morphometrics. To estimate the CS, we placed 11 landmarks and semilandmarks on the skull in dorsal view (for details, see fig. 1 and the appendix). Once landmarks were digitized, shape was analyzed using generalized Procrustes analysis (GPA; Bookstein 1991), and the CS was finally retrieved. The regression of CS on both body-length (Paul 2010) and body-size (Benson et al. 2014) estimates gives reassuringly high $R^{2}$ ( 0.83 and 0.93 , respectively; data not shown). We therefore empirically proved that CS is a very good proxy for body size in ceratopsian.

\section{Allometric Scaling}

We computed nonphylogenetic regressions (allometric scaling) of ornament complexity versus body size in all three clades. The allometric equation takes the form of a power function $Y=c M^{b}$, where $Y$ is the ornament complexity, $M$ is body mass, $c$ is the intercept, and $b$ is the scaling exponent. Our hypothesis is that $b$ is statistically equal to 0.25 for all clades.

The ornaments we dealt with are three-dimensional biological structures. Hence, to compute allometric scaling, we should ideally quantify complexity in the three dimensions and regress the complexity index versus body size.

For deer, we computed complexity as the logarithm of the sum of the Strahler numbers sSN over their antlers (Strahler 1957; fig. 1). For ceratopsians and ammonites, we computed the fractal dimension of the frill outer margin and suture, respectively, as the index of ornament complexity (fig. 1). This reduces the ornament structure to a one-dimensional length (actually an infinitely thin line of a given complexity). Hence, we regressed the complexity index against a one-dimensional body size index (the shell diameter and the centroid size for ammonites and ceratopsians, respectively) to maintain consistency between the allometric equations we computed for each clade.
The fractal dimension is a measure of the complication of a topological entity, describing its convolution at different scales of observation. For instance, an infinitely thin line has a topological dimension of 1 , yet its fractal dimension will be constrained between 1 and 2 in most real cases (Mandelbrot 1967). Using the fractal dimension changes a bit the formulation of the allometric relationship. Let us assume that we have an ammonite suture with a fractal length of $E=1$. The fractal dimension of the suture is the exponent $D$ of the power function

$$
E=N s^{D},
$$

where $N$ is the number of steps of length $s$ needed to cover the fractal length. If $D=1$, the fractal and topological lengths of the suture will both be 1 . For instance, with $D=1$, it takes 10 steps of length 0.1 to cover $E$. With $D=1.1$, the topological dimension will still be 1 , yet the fractal length will be 1.258 (because it takes $10^{-\log (0.1) \times 1.1}=$ 12.58 steps to cover the length $E$ ). With $D=1.2$, the fractal length will be 1.58 , and so on. From (1) it is easy to derive $D$ :

$$
-D=\frac{\log (N)}{\log (s)} .
$$

The fractal length could be equated to a given linear dimension $d$ of the biological object under scrutiny representing its size (such as the shell diameter of an ammonite; Zeide and Pfeifer 1991). Since we kept $s$ (the step length) constant in computing the fractal dimension, $-D \propto \log (N)$ over all ammonites and $\log (d) \propto \log (N)$ for any given shell. Therefore, the interspecific allometric scaling of fractal dimension in ammonites takes the form

$$
D=b \times \log (d)+c,
$$

where $\log (d)$ is the logarithm of the shell diameter. This formulation is correct insofar as the fractal length is a constant proportion of $d$ across species. This is most probably true of the homomorph ammonites we included in our analyses, where large species are satisfactorily represented as scaled-up versions of small species (meaning that overall body shape does not change with shell diameter). In ceratopsians, though, the frill grows disproportionately large with body size, so that small ceratopsians have relatively modest frills as compared to large species. The proportionality $-D \propto \log (N) \propto \log (d)$ can be rewritten as $-D \times k \propto$ $\log (d) \times k$, where $k=\log (x) / \log (d)$, which is the size ratio of the ornament size to the linear dimension $d$. If $k$ is invariant to size, the growth is isometric. From this formulation and equation (3), we derived the allometric scaling equation for ceratopsians frill:

$$
\frac{D \times \log \left(\mathrm{CS}_{\text {frill }}\right)}{\log \left(\mathrm{CS}_{\text {snout }}\right)}=b \times \log \left(\mathrm{CS}_{\text {frill }}\right)+c,
$$


where $\mathrm{CS}_{\text {frill }}$ and $\mathrm{CS}_{\text {snout }}$ represent the centroid size of the frill and the snout portions of the skull ( $\mathrm{CS}_{\text {snout }}$ is actually calculated from the tip of the snout to the frontoparietal suture), respectively (fig. 1).

Equations (3) and (4) respect dimensionality, since the fractal dimension was computed on a plane projection of the shell suture and frill outline for ammonites and ceratopsids, respectively, so that the fractal dimension $D$ is constrained between 1 and 2 (which is the fractal dimension of a convoluted line with topological dimension 1 ). The centroid size is the square root of summed squared distances; hence, its dimension is 1 as well.

\section{Phylogenetic Generalized Least Squares Regressions}

Our starting hypothesis is that the increase in complexity in ornament shapes is causally linked to increases in body size (i.e., to Cope's rule). To ensure this association was not due to phylogenetic effects, we applied phylogenetic generalized least squares (PGLS) regression (Grafen 1989) of body size on complexity for the three clades under scrutiny and tested several models of evolution. PGLS works by correcting the regression statistics assuming a given $\mathrm{co}^{-}$ variance matrix for the regression error term $\varepsilon$. Under the Brownian motion model, $\varepsilon$ is obtained from the tree variance/covariance matrix, which for any pair of species in the tree includes time from the tree root to the most recent common ancestor as an off-diagonal element and the distance from the root (for each species) as diagonal elements. The fit of the Brownian motion model to the tree and trait data could be contrasted against alternative models, based on tree transforms that modify the variance/covariance matrix (Pagel 1999). We used four alternative (to Brownian motion) models of evolution in PGLS. Under the $\lambda$ transform, the fit of the phylogeny to the data is maximized by transforming branch lengths in order to predict the covariance among trait values. Under $\delta$, the relative contribution of early (vs. late) evolution of the trait variance is estimated. The $\kappa$ model tests for the punctuational model of trait evolution. If $\kappa=0$, all branch lengths $l$ will be $l^{0}=1$, so that all of the phenotypic distance between any pair of species is independent of the time passed since their most recent common ancestor. Finally, under the trend model, a diffusion rate of trait evolution is tested so that all the phenotypic evolution across the tree is modeled to move away from the root state.

Since our trees were not ultrametric, before applying PGLS, we first estimated the variance vector (diagonal) of the transformed variance/covariance matrix according to each model of evolution. PGLS regressions were developed in $\mathrm{R}$ by using the package geiger (Harmon et al. 2008).

The results (including likelihood estimates and Akaike information criteria) are reported in table 1.

\section{Results}

\section{Deer Antlers}

The sSN is very high in antlers of Pleistocene and extant species such as the reindeer Rangifer tarandus, the stagmoose Cervalces scotti, the bush-antlered deer Eucladoceros dicranios, and species of the Megaloceros (Irish elk) clade (fig. 3). These species are well known for their exceptionally complex, heavily branching antlers (Geist 1971). The high sSN value is in agreement with the negative and significant regression between $\mathrm{sSN}$ and first appearance ages (adjusted $R^{2}=0.501$, slope $=-0.023, P \ll .01$ ). The phylogenetic signal of antler complexity is high and statistically greater than 0 (Blomberg's $K=0.85, P=.002$ ).

The relationship between stratigraphic duration and antler complexity is nonsignificant (slope $=-0.234$; 95\% confidence interval $[\mathrm{CI}]=-6.171-5.705 ; P=.937, R^{2}=$ $0.001, n=41)$. The same applies when phylogenetic correction is used (the best regression models are $\delta$ transform, $P=.774$, and the trend model, $P=.778$; table 1 ).

The relationship between antler complexity and body size (allometric scaling) is significant and positive, with the slope including the expected 0.25 value (slope $=0.346 ; 95 \%$ $\mathrm{CI}=0.201-0.449 ; P \ll .001, R^{2}=0.248, n=41$; fig. 2 ). PGLS regression of body size versus sSN is significant and positive under all evolutionary models (there is no model statistically superior to any other candidate model; see table 1).

\section{Ammonite Sutures}

We computed the fractal dimension of the ammonite sutures as an index of complexity (fig. 2). Among ammonite genera, we found the most complex sutures within Cretaceous genera of the Lytoceratina (Argonauticeras, Eulytoceras, Eogaudryceras, Ammonitoceras), late Jurassic to Cretaceous Phylloceratina (Adabofoloceras, Hyporbulites), and Cretaceous Ammonitina (Busnardoites, Desmoceras) all have a fractal dimension of $\sim 1.7$ (fig. 3). These results agree with the regression of ornament complexity versus first appearance ages (adjusted $R^{2}=0.611$, slope $=-0.0143, P \ll .01$ ). The phylogenetic signal of suture complexity is high and statistically greater than $0(K=0.87, P=.001)$.

The relationship between stratigraphic duration and suture complexity is significant and positive but weak (slope $=0.003 ; 95 \%$ CI $=0.002-0.004 ; P \ll .001, R^{2}=$ $0.094, n=241)$. Yet, such a relationship becomes nonsignificant when controlled for phylogeny (the best regression model is obtained by applying the $\lambda$ transform, $P=.781$; table 1).

Allometric scaling of suture complexity on shell size is significant and positive, with the slope very close to the expected 0.25 value $($ slope $=0.251 ; 95 \%$ CI $=0.178-0.324$; 
Table 1: Phylogenetic generalized least squares (PGLS) results of ornament complexity regressed against different candidate independent variables

\begin{tabular}{|c|c|c|c|c|}
\hline Clade, complexity, variable & Slope & $P$ & AIC & $\operatorname{logLik}$ \\
\hline \multicolumn{5}{|l|}{ Deer: } \\
\hline \multicolumn{5}{|l|}{ Body size: } \\
\hline $\mathrm{BM}$ & 8.993378 & .023485 & 272.9249 & -133.462 \\
\hline$\lambda$ & 9.16526 & .015648 & 272.2581 & -133.129 \\
\hline$\delta$ & 8.336953 & .01579 & 271.7321 & -132.866 \\
\hline$\kappa$ & 9.002847 & .025669 & 272.7969 & -133.399 \\
\hline Trend & 8.110466 & .014581 & 271.9113 & -132.956 \\
\hline \multicolumn{5}{|l|}{ Duration: } \\
\hline BM & .638729 & .756253 & 279.411 & -136.706 \\
\hline$\lambda$ & .596888 & .787489 & 279.259 & -136.63 \\
\hline$\delta^{\mathrm{a}}$ & .631165 & .774093 & 278.5337 & -136.267 \\
\hline$\kappa$ & .628829 & .760907 & 279.1527 & -136.576 \\
\hline Trend $^{\mathrm{a}}$ & .634484 & .778472 & 278.7307 & -136.365 \\
\hline \multicolumn{5}{|l|}{ Ammonites: } \\
\hline \multicolumn{5}{|l|}{ Body size: } \\
\hline $\mathrm{BM}$ & .038592 & .051563 & -365.658 & 185.8291 \\
\hline$\lambda$ & .024002 & .243451 & -402.222 & 204.111 \\
\hline$\delta$ & .031611 & .107647 & -364.981 & 185.4905 \\
\hline$\kappa^{\mathrm{a}}$ & .060515 & .004321 & -405.012 & 205.5059 \\
\hline Trend & .034201 & .082966 & -365.626 & 185.8128 \\
\hline \multicolumn{5}{|l|}{ Duration: } \\
\hline $\mathrm{BM}$ & -.03496 & .020292 & -366.712 & 186.3562 \\
\hline$\lambda^{\mathrm{a}}$ & .00529 & .780904 & -400.776 & 203.388 \\
\hline$\delta$ & -.03386 & .020255 & -367.198 & 186.5988 \\
\hline$\kappa$ & -.00282 & .884394 & -396.677 & 201.3384 \\
\hline Trend & -.03418 & .021546 & -367.346 & 186.6732 \\
\hline \multicolumn{5}{|l|}{ Ceratopsians: } \\
\hline \multicolumn{5}{|l|}{ Body size: } \\
\hline $\mathrm{BM}$ & .07282 & .24711 & -68.2079 & 37.1039 \\
\hline$\lambda$ & .15365 & .00586 & -79.5213 & 42.7607 \\
\hline$\delta$ & .07085 & .27876 & -76.4315 & 41.2157 \\
\hline$\kappa^{\mathrm{a}}$ & .15563 & .00342 & -86.8448 & 46.4224 \\
\hline Trend & .07387 & .24003 & -74.6392 & 40.3196 \\
\hline
\end{tabular}

Note: For each group, we report the regression stats as the first row and the fitted values of the regression parameters under different models of trait evolution. $\log$ Lik $=\log$ likelihood. $\mathrm{BM}=$ Brownian motion.

${ }^{a}$ The best PGLS regression model (in terms of Akaike information criterion [AIC]).

$\left.P \ll .001, R^{2}=0.167, n=241\right)$. The PGLS regression between body size and complexity is significant and positive under the $\kappa$ transform (which gives the best regression model, $P=.004$; table 1$)$.

\section{Ceratopsian Frill}

We computed the fractal dimension of the frill outer margin as an index of complexity (fig. 2). In ceratopsians, the largest and most elaborate frills belong to late Cretaceous chasmosaurines (Kosmoceratops richardsoni, Torosaurus latus, and Vagaceratops irvinensis) and centrosaurines (Styracosaurus albertensis, Centrosaurus apertus, and Diabloceratops eatoni), thus confirming that frill complexity increases in more recent species (complexity vs. first appearance ages regression: adjusted $R^{2}=0.196$, slope $\left.=-0.001, P=.005\right)$. The highest values of the fractal dimension of the frill margin are found in Kosmoceratops, Styracosaurus, Diabloceratops, and Centrosaurus (fig. 3). The latter present fractal dimension between 1.1 and 1.2, up to nearly 1.3 in Kosmoceratops. The evolution of the fractal dimension of the frill margin shows a strong and significant phylogenetic signal $(K=1.01, P=.01)$. The relationship between the fractal dimension of the frill margin and skull centroid size is significant and positive (slope $=0.076 ; 95 \% \mathrm{CI}=0.043$ $-0.108 ; P \ll .001, R^{2}=0.419, n=34$ ), as is the relationship between the fractal dimension and centroid size of the snout portion of the skull (slope $=0.098 ; 95 \%$ CI $=0.061$ 

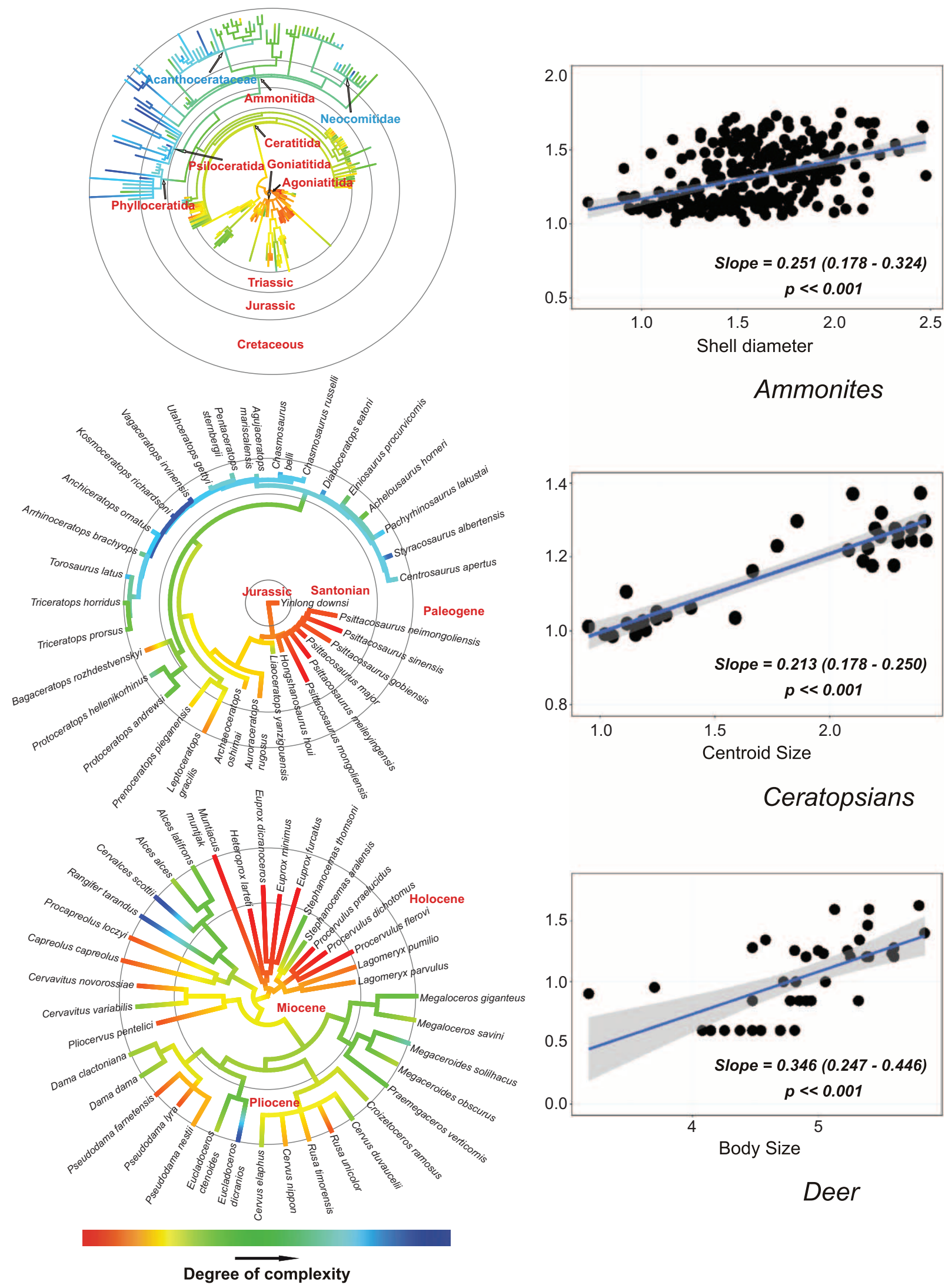

Ammonites

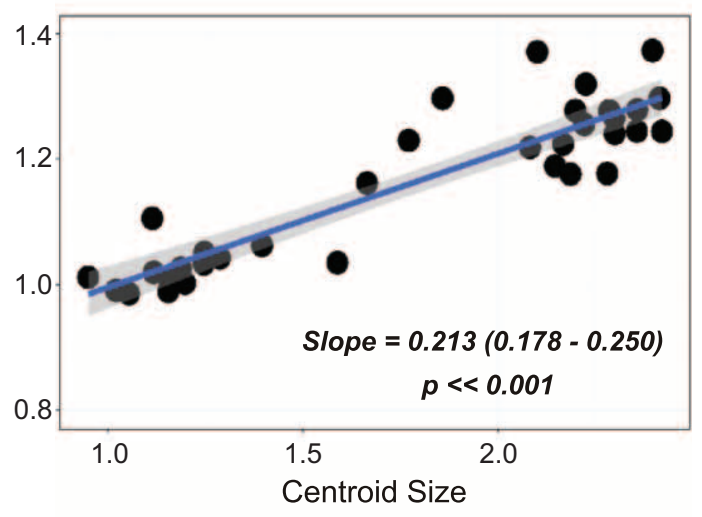

Ceratopsians

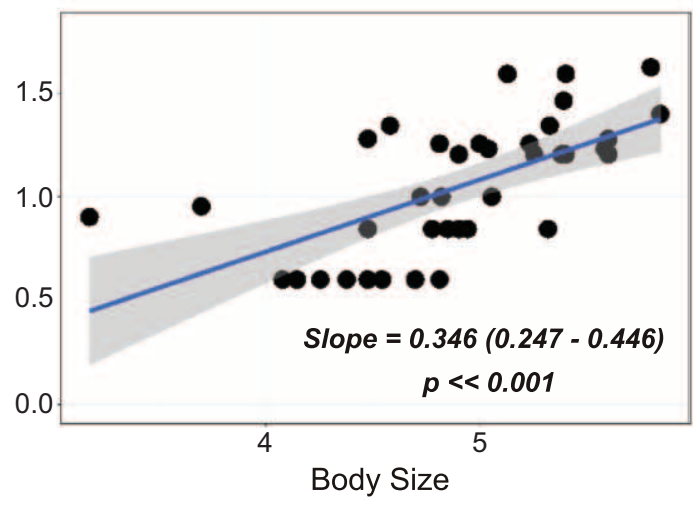

Deer

Figure 3: Ornament evolution and scaling. Left, the phylogenetic trees of the clades considered here are plotted. The color of the branches represents the degree of ornament complexity, reconstructed according to the parsimony method in Mesquite. To the right of each tree are 
$\left.-0.137 ; P \ll .001, R^{2}=0.457, n=34\right)$. Yet the relationship between frill and snout centroid sizes is significant and positive $($ slope $=1.306 ; 95 \% \mathrm{CI}=1.232-1.380 ; P \ll .001$, $\left.R^{2}=0.975, n=34\right)$. The slope of this relationship is statistically $>1$, which means the frill grows faster than snout size. This makes correcting for allometric scaling (according to eq. [4]; see "Material and Methods") necessary. Allometric scaling of ornament complexity against frill size is significant, positive, and, crucially, does not differ significantly from 0.25 (slope $=0.213 ; 95 \%$ CI $=0.178-0.250$; $P \ll .001, R^{2}=0.814, n=34$; fig. 2). Allometric scaling of size-corrected ornament complexity against frill size is significant under the $\kappa$ model $(P=.003$; table 1$)$.

We compared the allometric scaling regressions of ornament complexity for the three groups by means of analysis of variance. The slopes of the three clades are not significantly different from one another $(F=1.771, P=.180)$. None of the pairwise differences among clades are statistically significant.

\section{Discussion}

Our data come from different sources, pertain to clades that are phylogenetically distant and ecologically dissimilar, and span more than 420 million years of the evolution of life, from Devonian ammonites to extant deer. We covered in our data $>80 \%$ of known ceratopsians for which nondeformed skulls are known, whereas the fossil record of deer species and, especially, ammonite genera is much more abundant than we could have reasonably covered in our data. The role of sexual selection on elaboration of traits examined in the three clades differs, from strong for antlers in deer to nonexistent in the complexity of ammonite sutures.

Despite all of these remarkable dissimilarities, allometric scaling exponents of complexity are statistically indistinguishable from one another. Most importantly, they are statistically no different from the scaling exponent of developmental time on body mass (i.e., 0.25; Roff 2002), as we expected (fig. 1). Confidence intervals around the slope estimates are rather large. This was expected under our hypothesis of unaltered rate of complexity increase between the ontogenies of each species and its evolutionary descendant. Yet, although deviations from this crude assumption provide noise around the estimates, the degree of this variation does not affect critically the three exponents we computed, thus confirming our null hypothesis.

The theory for ornament evolution we tested here was based on the observation that ornament complexity increases with body size during ontogeny in all of the three clades we analyzed (Goss 1983; Sampson 2001). We reasoned that if the developmental rate holds constant in ancestor/descendant relationships, then the complexity of the ornament should increase over time by virtue of extended (absolute) growth in larger (and geologically younger) species' ontogenies. The increase in ornament complexity would be then related to the general tendency for body size to increase over time along lineages (namely, Cope's rule; Alroy 1998; Raia et al. 2012). Therefore, subadults of a large derived species should resemble, in terms of ornament complexity, the fully grown representatives of smaller, geologically older individuals of the same clade. In red deer, young male have fewer tines in their antlers than mature stags do (typically, 3-4 tines), similar to adult chital or sambar deer. This is true for centrosaurines as well, making it difficult to assign young individuals to particular species (Sampson et al. 1997). García-Ruiz et al. (1990) showed that ammonite suture complexity increases with the age of the individual. In fact, it has been proposed that the increase in complexity of suture lines results from covariation with increasing adult size (Monnet et al. 2011). Crucially, consistent with our theory, the smaller-bodied descendants of large, highly ornamented species should have less complex ornaments. For instance, late-Pleistocene dwarf insular deer Cervus from Crete had proportionally large, but very simple antlers with 2 tines only, although their ancestors had 14 or more tines (De Vos 1984). The same holds true for the extinct Sardinian island deer Praemegaceros cazioti (Caloi and Palombo 1995) and the Ryukyus deer Cervus astylodon (Matsumoto and Otsuka 2000).

Stanley (1979) posited that increases in biological complexity should co-occur with higher extinction rate and larger body size. This common trend would configure as Cope's original law of the unspecialized (i.e., the idea that clades start with small, simple, and unspecialized representatives; Raia and Fortelius 2013). Support for this "law" is controversial. Body size (Cope's rule) and concomitant complexity increase was reported in all the groups we stud-

linear regression plots between the complexity index of the ornamental trait and the body size for each clade. For ammonites, we calculated the linear regression between the mean fractal dimension of the suture line per genus and the logarithm of the mean shell diameter per genus. For ceratopsians, we calculated the linear regression between the fractal dimension of the frill outer margin and the skull centroid size, which is a body size index retrieved by applying geometric morphometric techniques. For deer, we calculated the linear regression between the log of the sum of the Strahler number ( $\mathrm{sSN}$ ) and the logarithm of the estimated body size. The gray area in the plots represents the confidence intervals around the estimates. 
ied here. As we commented elsewhere (Raia et al. 2012), Cope's rule applies because of a trend for increased specialization during the clades history, which, in turn, is spurred by major tectonic events and changes in climate (Raia et al. 2012). In fact, hoofed mammal species bearing elaborate weaponry and ornaments radiated during moments of intense climate change (Prothero and Schoch 2002). The common explanation is that transitions from forested to open habitats likely increased group size and, hence, the potential for males to access multiple females (Janis 1982; Sampson 1997). However, derived, large-sized ammonite genera with complex sutures persist as long in the fossil record as small, simple-sutured genera (Boyajian and Lutz 1992). In mammals, species belonging to lineages showing higher extinction rate are not those showing the most elaborate ornaments (Raia et al. 2011). In fact, we found that ornament complexity is unrelated to species longevity in the fossil record of ammonites and deer (see above).

Our results support the existence of a close relationship between body size and ornament complexity but not between complexity and reduced duration in the fossil record. Regarding complexity, we show that it correlates to body size alone, even when phylogenetic effects are controlled for. This supports our hypothesis that complexity scales to the one-quarter power of body size.

We found strong evidence that the familiar evolution of ornaments toward increased complexity (Stanley 1979; Bonner 1988) is the passive consequence of selection for larger body size, which is Cope's rule. In fact, while ammonite sutures certainly did not develop to attract mates or to defy opponents and predators, their evolution is statistically consistent with that of deer and frilled ceratopsians.

\section{Conclusion}

Although the evolution of ornament size is clearly driven by sexual selection (Kodric-Brown et al. 2006), the evolution of ornaments' complexity (hence their shape) is a consequence of the common trend for increased body size within lineages, which is Cope's rule. Thus, ornament complexity scales to the universal scaling exponent of biological times to body size. Whether or not ornaments are used in mate choice or male-male competition or have a dual function, it is probably their size rather than their shape that is acted upon by sexual selection.

\section{Acknowledgments}

All the data used to produce the statistical analyses presented here are available as supplementary material. We are grateful to many institutions and their staff for allowing access to the collections in their care and for pro- viding precious help in collecting specimen pictures. We are grateful to G. Sansalone, who collected photographs of Triceratops prorsus (BSPG 1964-I-458) at the Bayerische Staatssammlung für Paläontologie und Historische Geologie, Munich, Germany. We similarly thank A. H. van Heteren, who secured photographs of Triceratops horridus (MNHN F1912.20) from the Muséum National d'Histoire Naturelle, Paris, France. We also want to thank A. A. Farke for useful suggestions.

\section{Literature Cited}

Abràmoff, M. D., P. J. Magalhães, and S. J. Ram. 2004. Image processing with ImageJ. Biophotonics International 11:36-42.

Alroy, J. 1998. Cope's rule and the dynamics of body mass evolution in North American fossil mammals. Science 280:731-734.

Andersson, M. 1994. Sexual selection. Princeton University Press, Princeton, NJ.

Benson, R. B., N. E. Campione, M. T. Carrano, P. D. Mannion, C. Sullivan, P. Upchurch, and D. C. Evans. 2014. Rates of dinosaur body mass evolution indicate 170 million years of sustained ecological innovation on the avian stem lineage. PLoS Biology 12: e1001853.

Bergstrom, C. T., and L. A. Real. 2000. Toward a theory of mutual mate choice: lessons from two-sided matching. Evolutionary Ecology Research 2:493-508.

Bonduriansky, R. 2007. Sexual selection and allometry: a critical reappraisal of the evidence and ideas. Evolution 61:838-849.

Bonner, J. T. 1988. The evolution of complexity by means of natural selection. Princeton University Press, Princeton, NJ.

Bookstein, F. L. 1986. Size and shape spaces for landmark data in two dimensions. Statistical Science 1:181-242.

- 1991. Morphometric tools for landmark data: geometry and biology. Cambridge University Press, Cambridge.

Boyajian, G., and T. Lutz. 1992. Evolution of biological complexity and its relation to taxonomic longevity in the Ammonoidea. Geology 20:983-986.

Caloi, L., and M. R. Palombo. 1995. Il megacerino di Dragonara: interpretazione funzionale di alcuni caratteri cranici. Geologica Romana 31:29-40.

Caro, T. M., C. M. Graham, C. J. Stoner, and M. M. Flores. 2003. Correlates of horn and antler shape in bovids and cervids. Behavioral Ecology and Sociobiology 55:32-41.

Darwin, C. 1871. The descent of man. W. Clowes, London.

De Vos, J. 1984. The endemic Pleistocene deer of Crete. NorthHolland, Amsterdam.

Dodson, P., C. A. Forster, and S. D. Sampson. 2004. Ceratopsidae. Pages 494-513 in D. B. Weishampel, P. Dodson, and H. Osmólska, eds. The Dinosauria. University of California Press, Berkeley.

Dommergues, J. L. 1990. Ammonoids. Pages 162-187 in K. J. McNamara, ed. Evolutionary trends. Belhaven, New York.

Emlen, D. J. 2008. The evolution of animal weapons. Annual Review of Ecology, Evolution, and Systematics 39:387-413.

Emlen, D. J., I. A. Warren, A. Johns, I. Dworkin, and L. C. Lavine. 2012. A mechanism of extreme growth and reliable signaling in sexually selected ornaments and weapons. Science 337:860-864.

García-Ruiz, J. M., A. Checa, and P. Rivas. 1990. On the origin of ammonite sutures. Paleobiology 16:349-354. 
Geist, V. 1971. The relation of social evolution and dispersal in ungulates during the Pleistocene, with emphasis on the Old World deer and the genus Bison. Quaternary Research 1:285-315.

- 1998. Deer of the world: their evolution, behaviour and ecology. Stackpole, Mechanicsburg, PA.

Goss, R. J. 1983. Deer antlers: regeneration, function and evolution. Academic Press, New York.

Grafen, A. 1989. The phylogenetic regression. Philosophical Transactions of the Royal Society B: Biological Sciences 326:119-157.

Harmon, L. J., J. T. Weir, C. D. Brock, R. E. Glor, and W. Challenger. 2008. GEIGER: investigating evolutionary radiations. Bioinformatics 24:129-131.

Holtz, T. R., Jr. 2007. Dinosaurs: the most complete, up-to-date encyclopedia for dinosaur lovers of all ages. Random House, London.

Hone, D. W. E., D. Naish, and I. C. Cuthill. 2012. Does mutual sexual selection explain the evolution of head crests in pterosaurs and dinosaurs? Lethaia 45:139-156.

Horner, J. R., and M. B. Goodwin. 2006. Major cranial changes during Triceratops ontogeny. Proceedings of the Royal Society B: Biological Sciences 273:2757-2761.

. 2009. Extreme cranial ontogeny in the Upper Cretaceous dinosaur Pachycephalosaurus. PLoS ONE 4:e7626.

Janis, C. M. 1982. Evolution of horns in ungulates: ecology and paleoecology. Biological Reviews 57:261-318.

Karperien, A. 1999-2013. FracLac for ImageJ, version 2.5. http://rsb .info.nih.gov/ij/plugins/fraclac/FLHelp/Introduction.htm.

Knell, R. J., D. Naish, J. L. Tomkins, and D. W. Hone. 2013. Sexual selection in prehistoric animals: detection and implications. Trends in Ecology and Evolution 28:38-47.

Kodric-Brown, A., R. M. Sibly, and J. H. Brown. 2006. The allometry of ornaments and weapons. Proceedings of the National Academy of Sciences of the USA 103:8733-8738.

Kokko, H., and R. A. Johnstone. 2002. Why is mutual mate choice not the norm? operational sex ratios, sex roles and the evolution of sexually dimorphic and monomorphic signalling. Philosophical Transactions of the Royal Society B: Biological Sciences 357:319330

Kuijper, B., I. Pen, and F. J. Weissing. 2012. A guide to sexual selection theory. Annual Review of Ecology, Evolution, and Systematics 43:287-311.

Maddison, W. P., and D. R. Maddison. 2001. Mesquite: a modular system for evolutionary analysis. Version 2.74 . http://mesquiteproject .org.

Mandelbrot, B. B. 1967. How long is the coast of Britain? statistical self-similarity and fractional dimension. Science 156:636-638.

Matsumoto, Y., and H. Otsuka. 2000. Morphotypes of fossil deer (Cervus astylodon) and its miniaturization process in the Ryukyu Islands. Tropics 10:155-164.

Møller, A. P., and T. R. Birkhead. 1994. The evolution of plumage brightness in birds is related to extrapair paternity. Evolution 48 : 1089-1100.

Monnet, C., K. De Baets, and C. Klug. 2011. Parallel evolution controlled by adaptation and covariation in ammonoid cephalopods. BMC Evolutionary Biology 11:115.

Padian, K., and J. R. Horner. 2011. The evolution of "bizarre structures" in dinosaurs: biomechanics, sexual selection, social selection or species recognition? Journal of Zoology 283:3-17.
2013. Misconceptions of sexual selection and species recognition: a response to Knell et al. and to Mendelson and Shaw. Trends in Ecology and Evolution 28:249-250.

- 2014. The species recognition hypothesis explains exaggerated structures in non-avialan dinosaurs better than sexual selection does. Comptes Rendus Palevol 13:97-107.

Pagel, M. 1999. Inferring the historical patterns of biological evolution. Nature 401:877-884.

Paul, G. S. 2010. The Princeton field guide to dinosaurs. Princeton University Press, Princeton, NJ.

Prothero, D. R., and R. M. Schoch. 2002. Horns, tusks, and flippers: the evolution of hoofed animals. Johns Hopkins University Press, Baltimore.

Raia, P., F. Carotenuto, J. T. Eronen, and M. Fortelius. 2011. Longer in the tooth, shorter in the record? the evolutionary correlates of hypsodonty in Neogene ruminants. Proceedings of the Royal Society B: Biological Sciences 278:3474-3481.

Raia, P., F. Carotenuto, F. Passaro, D. Fulgione, and M. Fortelius. 2012. Ecological specialization in fossil mammals explains Cope's rule. American Naturalist 179:328-337.

Raia, P., and M. Fortelius. 2013. Cope's law of the unspecialized, Cope's rule, and weak directionality in evolution. Evolutionary Ecology Research 15:747-756.

Revell, L. J. 2012. Phytools: an R package for phylogenetic comparative biology (and other things). Methods in Ecology and Evolution 3:217-223.

Roff, D. A. 2002. Life history evolution. Sinauer, Sunderland, MA.

Sampson, S. D. 1997. Dinosaur combat and courtship. Pages 383-393 in M. K. Brett-Surman, T. R. Holtz, and J. O. Farlow, eds. The complete dinosaur. Indiana University Press, Bloomington.

2001. Speculations on the socioecology of ceratopsid dinosaurs (Ornitnischia: Neoceratopsia). Pages 263-276 in D. H. Tanke, K. Carpenter, and M. W. Skrepnick, eds. Mesozoic vertebrate life. Indiana University Press, Bloomington.

Sampson, S. D., M. J. Ryan, and D. H. Tanke. 1997. Craniofacial ontogeny in centrosaurine dinosaurs (Ornithischia: Ceratopsidae): taxonomic and behavioral implications. Zoological Journal of the Linnean Society 121:293-337.

Santana, S. E., J. L. Alfaro, and M. E. Alfaro. 2012. Adaptive evolution of facial colour patterns in Neotropical primates. Proceedings of the Royal Society B: Biological Sciences 279:2204-2211.

Stanley, S. M. 1973. An explanation for Cope's rule. Evolution 27:126.

-1979. Macroevolution. Freeman, San Francisco.

Strahler, A. N. 1957. Quantitative analysis of watershed geomorphology. Civil Engineering 101:1258-1262.

Stuart-Fox, D. M., and T. J. Ord. 2004. Sexual selection, natural selection and the evolution of dimorphic coloration and ornamentation in agamid lizards. Proceedings of the Royal Society B: Biological Sciences 271:2249-2255.

West-Eberhard, M. J. 1983. Sexual selection, social competition, and speciation. Quarterly Review of Biology 58:155-183.

Zeide, B., and P. Pfeifer. 1991. A method for estimation of fractal dimension of tree crowns. Forest Science 37:1253-1265.

Associate Editor: David N. Reznick Editor: Judith L. Bronstein 